\title{
Factors associated with mortality in patients with tuberculosis
}

David J Horne ${ }^{1 *}$, Rebecca Hubbard ${ }^{2,3}$, Masahiro Narita ${ }^{1,4,5}$, Alexia Exarchos ${ }^{6}$, David R Park ${ }^{1}$, Christopher H Goss ${ }^{1}$

\begin{abstract}
Background: Known risk factors for death following a diagnosis of tuberculosis may not be applicable to current U.S. cases. We evaluated the factors associated with all-cause mortality in patients with tuberculosis in Washington State.

Methods: Using data from the Tuberculosis Information Management System of Washington State, we conducted a cohort study of all residents diagnosed with tuberculosis from 1993 through 2005. Death from any cause was ascertained through the Washington State Death Certificate Data Files. Proportional hazards models were used to estimate the independent effect on all-cause mortality of demographic, clinical, and behavioral characteristics.
\end{abstract}

Results: During a median follow-up of 6 years in 3451 patients treated for tuberculosis, there were 417 deaths. Mortality was independently associated with increasing age, male gender, HIV-coinfection, and U.S. birth. Within 1 year of tuberculosis diagnosis, treatment by a private provider and the use of directly observed therapy were also independently associated with increased mortality. In addition, an interaction term of private provider times directly observed therapy was also significantly associated with mortality.

Conclusions: We identified factors independently associated with increased all-cause mortality following a diagnosis of tuberculosis. The associations between mortality and provider type should be evaluated with more thorough adjustment for severity of illness, but suggest important directions for future research.

\section{Background}

There were over 1.7 million deaths due to tuberculosis (TB) worldwide in 2007 with a global case-fatality of 19\% [1,2]. The United States TB-specific case-fatality has been estimated at $5 \%$ or less in recent years, although a Baltimore-based study observed a case-fatality of $24 \%$ in sputum smear positive patients while on treatment $[2,3]$. Studies evaluating risk factors for death following a diagnosis of TB have typically focused on specific patient populations (e.g. patients with multidrug resistant-TB (MDR-TB), human immunodeficiency virus (HIV)-coinfected patients, and patients enrolled in TB treatment trials) [4-8]. Population-based studies have identified a number of risk factors including HIV-coinfection, non-use of directly observed therapy (DOT), care from a physician inexperienced with TB management, drug resistance, and more than one site of TB

\footnotetext{
* Correspondence: dhorne@u.washington.edu

'Department of Medicine, University of Washington School of Medicine,

Seattle, WA, USA

Full list of author information is available at the end of the article
}

involvement as associated with death following a diagnosis of TB [7,9-11].

The applicability of these prior studies to the U.S. is unknown as they were conducted outside of the United States or during the TB resurgence of the 1990s. The current characteristics of TB cases and TB control activities in the U.S. include increased use of DOT, increased proportion of $\mathrm{TB}$ due to foreign born persons, and greater delivery of TB treatment by public health departments; Washington State has mirrored these trends [12]. We performed a population-based study of factors associated with mortality following a diagnosis of TB in Washington State during a period of decreasing TB incidence and stable treatment recommendations [13]. We examined demographic, clinical and treatment related characteristics associated with survival. Some of the results of this study have been previously reported in the form of an abstract [14]. 


\section{Methods \\ Study Population}

The population in this cohort study consisted of all individuals diagnosed with active $\mathrm{TB}$ and reported to the Washington State Department of Health Tuberculosis Registry through the TB Information Management System (TIMS) during the period of January 1, 1993 through December 31, 2005. Tuberculosis is a reportable disease and the completeness of reporting in Washington State is estimated to be nearly 100 percent [15]. TB cases were defined according to the Centers for Disease Control and Prevention (CDC) guidelines [16]. The Washington State Institutional Review Board approved all study protocols and analyses.

\section{Data Collection}

We merged data from two sources: 1) TIMS, and 2) the Washington State Death Certificate Data Files. The Report of Verified Case of TB (RVCT) is the national TB surveillance form produced by the CDC. Local public health jurisdictions in Washington State are required to complete and submit the RVCT to the State, where information is entered into TIMS. Data on deaths in Washington State and deaths of Washington State residents who died in other states are maintained in the Death Certificate Files. We obtained death registry information from January 1, 1993 to December 31, 2005. First and last names, as well as middle initials and home address when available, were used in conjunction with birth dates to link study subjects with data from the death registry.

Person-time of cohort members began when antituberculosis treatment was started and ended on the date of death or December 31, 2005, whichever came first (information on the timing of the index culture was available only for patients with pulmonary TB). We decided on the variables for our model a priori based on known and potential risk factors, including: subject characteristics (age, gender, race/ethnicity, HIV status, U.S. birth, residence, substance use, income), disease characteristics (site of TB disease, radiographic cavitary disease, drug susceptibility, prior TB), and factors related to treatment (provider type, use of DOT). These factors were defined according to the RVCT [17]. Race and ethnicity were based on self-report. Provider type was defined as a health department if outpatient care was provided by a state or local health department. DOT was defined as treatment ingestion observed by a health care provider or other reliable person [17]. Variables available for the analysis that were not defined by the RVCT included recency of immigration to the United States and median household income. The latter was based on U.S. census data from 2000 using the subjects' home zip code. Extrapulmonary TB was defined as disease in organs other than the lungs or pleura [7]. Pulmonary TB with cavitation was defined as the presence of cavitary lesions on chest radiograph. In order to assess for temporal affects on survival, we included a variable based on diagnosis in the first half or last half of the study period ("year of diagnosis after 1999").

In the bivariate and multivariable analyses, we made a number of assumptions. Provider type was categorized as private provider if TB care was never received from a health department; otherwise care was categorized as a health department. For example, a subject who was discharged from a hospital and received treatment through a health department would be categorized as having a provider type of health department. Therapy type was categorized as self-administered or "other" (including DOT only or a combination of the two). Because of limited numbers, Pacific Islanders/Hawaiians were included in the Asian category. If information was missing regarding alcohol abuse, then patients were classified as non-abusers. Similar assumptions were made for injection drug use, homelessness and institutional residence. Subjects were considered HIV-positive only if they were known to be seropositive. Patients without drug susceptibility results were classified as having susceptible isolates.

\section{Statistical Analysis}

We used Cox proportional hazards models to analyze associations between candidate risk factors and survival. The proportional hazards assumption was tested using graphical inspection and tests for time trends of Schoenfeld residuals. Variables that did not satisfy the proportional hazards assumption (income, health care provider type, and therapy type), were modelled after being stratified by time. We also examined the significance of two-way interactions between these timestratified variables, in addition to interactions between age and sex. All of our pre-selected variables were included in the final multivariable models based on our initial hypotheses. To examine the effect of missing data and our assumptions on the model, we analyzed the data: 1) after excluding patients with missing data, and 2) imputing values for missing data based on frequencies observed for subjects with non-missing data. After observing the relationship between provider type and survival, we conducted a simulation study to assess the potential impact of differential loss to follow-up among subjects by provider type (see additional file 1). Statistical analyses were performed using Stata 10 (StataCorp, College Station, TX) and R 2.2.0 (R Foundation for Statistical Computing, Vienna, Austria). 


\section{Results}

During the 13-year study period, 3451 persons with active TB were diagnosed in Washington State. The median follow-up period was 5.9 years (interquartile range [IQR]: 2.6-9.3). There were 417 deaths and cumulative all-cause mortality at years 1 and 3 were $4.6 \%$ (95\% confidence interval [CI] 3.9-5.4) and 7.7\% (CI 6.88.8), respectively. Among HIV-coinfected patients the one-year and three-year mortality were $10.5 \%$ (CI 6.417.2 ) and $18.9 \%$ (CI 12.8-27.8), respectively.

The mean $( \pm$ SD) age at the time of diagnosis was 45 $( \pm 21)$ years. The date of initial sputum culture positivity was reported in 1568 patients (68\% of the pulmonary TB cases), and anti-tuberculosis therapy was started less than one week after collection of the initial sputum culture in $70 \%$. The median time to treatment initiation in these 1568 patients was 2 days after sputum collection (IQR: $0-12$ ). In the entire cohort, $85 \%$ of patients were documented as completing therapy, $3 \%$ moved prior to completion of treatment, $2 \%$ of patients were considered lost and $1 \%$ refused treatment; among the remaining patients, $5 \%$ died, $1 \%$ had "other" as the stop reason, and information was missing in 3\%.

Baseline characteristics for the cohort are in Table 1. Most subjects were male (61\%), foreign born (63\%), received DOT (61\%), and were treated exclusively by a public health department (54\%). There was a low prevalence of known HIV-coinfection (5\%). Except for resistance to isoniazid (8\%) and streptomycin (6.7\%), there was less than $2 \%$ prevalence of resistance to any single drug; the prevalence of MDR-TB was $1 \%$. Mortality was lowest in females and in persons who were younger, non-Caucasian, HIV-negative, and foreign born, and also in persons without a prior history of TB or alcohol abuse. The presence of both pulmonary and extrapulmonary diseases was associated with an elevated death rate, as was receipt of care through a private provider.

In the multivariable model that included all hypothesized risk factors, we found that increasing age, male gender, HIV-coinfection, treatment by a private health care provider, the use of DOT, U.S. birth, and not being a recent immigrant were independently associated with increased mortality (Table 2). In developing our Cox proportional hazards model, income, health care provider type, and therapy type did not satisfy assumptions of proportional hazards. However, after stratifying by before or after the first year of follow-up, these variables satisfied the proportional hazards assumption. Treatment by a private health care provider (HR 5.1; $95 \% \mathrm{CI}$, 3.5-7.3) and use of DOT (HR 3.0; 95\% CI, 1.9-4.5) were associated with death only within one year of the diagnosis of TB; patients who survived one year were no longer at increased risk of death based on these variables. Income, after time stratification, was no longer associated with survival. There were no significant interactions between gender and age.

We evaluated our time stratified variables by modelling an interaction between health care provider type and therapy type (Table 3). Different from our final model, we found that receiving treatment from a private health care provider was significantly associated with decreased survival during the first year after a diagnosis of TB regardless of therapy type, although the strength of the association was greater if DOT was used (HR 8.5; 95\% CI, 4.3-17.1) than if treatment was solely self-administered (HR 2.2; 95\% CI, 1.1-4.6).

TB-related deaths were $21 \%$ of all deaths and $39 \%$ of the deaths that occurred within 12 months of starting TB treatment. We assessed the effect on the model of limiting mortality to TB-related deaths, defined as a TBrelated International Classification of Diseases code listed under the multiple contributing causes of death on the death certificate. Increasing age and HIV-coinfection were associated with a TB-related death; within one year of TB treatment initiation, treatment by a private health care provider and use of DOT were also associated with a TB-related death (see additional file 2).

As differences in illness severity could introduce confounding if a subject's death and TB diagnosis occurred during the same hospitalization, we assessed the effects on our model of excluding subjects who died within 30 -days of a diagnosis of TB $(n=92)$. Although the HR associated with treatment by a private health care provider was attenuated, it remained elevated (HR 2.8, 1.7-4.6; see additional file 3). In order to assess the impact of missing data and our assumptions on our models, we repeated our analyses after: 1) excluding patients with missing data, and 2) using imputed values for missing data. There were no significant changes in the observed associations compared to our final model (results not shown).

We evaluated the association of sputum smear positivity and survival in patients with pulmonary TB. Neither sputum smear positivity nor cavitation on chest radiographs was found to be associated with survival (see additional file 4). In patients with date of initial culture collection, a delay in treatment (defined as treatment initiation more than one week after sputum collection) was not associated with death in bivariate or multivariable analysis.

\section{Discussion}

All-cause mortality in our study was predicted by older age, HIV-coinfection, male gender, U.S. birth, remote immigration, care through a private health care provider and the use of DOT. The association between care 
Table 1 Person-years, deaths and death rates by selected baseline characteristics

\begin{tabular}{|c|c|c|c|c|}
\hline Characteristics & N (\%) (total = 3451) & Person- years & Mortality Rate & $95 \% \mathrm{Cl}$ \\
\hline Age, years: median (SD) & $45(21)$ & & & \\
\hline \multicolumn{5}{|l|}{ Gender } \\
\hline Female & $1349(39.1)$ & 8183 & 12.3 & $10.2-15.0$ \\
\hline Male & $2102(60.9)$ & 12585 & 21.7 & $19.3-24.4$ \\
\hline \multicolumn{5}{|l|}{ Race } \\
\hline Asian/Pacific Islander & $1321(38.2)$ & 8159 & 10.3 & 8.3-12.8 \\
\hline Black & $438(12.7)$ & 2316 & 13.4 & $9.4-19.0$ \\
\hline Caucasian & $982(28.5)$ & 5859 & 35.2 & $30.7-40.3$ \\
\hline Latino & $502(14.5)$ & 3097 & 7.8 & $5.2-11.6$ \\
\hline Native American & $179(5.2)$ & 1093 & 23.8 & $16.2-34.9$ \\
\hline Unknown & $29(0.8)$ & 204 & 14.7 & $4.7-45.6$ \\
\hline \multicolumn{5}{|l|}{ HIV Status } \\
\hline Negative & $1976(57.2)$ & 11753 & 11.7 & 9.9-13.8 \\
\hline Positive & $166(4.8)$ & 905 & 44.2 & $32.4-60.2$ \\
\hline Unknown & 1309 (37.9) & 8109 & 24.2 & $21.1-27.9$ \\
\hline \multicolumn{5}{|l|}{ Major site of disease } \\
\hline Pulmonary/pleural & $2652(76.8)$ & 16134 & 18.6 & $16.6-20.8$ \\
\hline Lymphatic & $425(12.3)$ & 2459 & 11.0 & $7.5-16.0$ \\
\hline Bone-joint/GU/Peritoneal & $206(5.9)$ & 1264 & 19.8 & $13.4-29.3$ \\
\hline Miliary/Meningeal & $92(2.7)$ & 515 & 33.0 & $20.5-53.1$ \\
\hline Other & $76(2.2)$ & 395 & 5.3 & $5.3-30.4$ \\
\hline \multicolumn{5}{|l|}{ Site of disease } \\
\hline Pulmonary & $2288(66.3)$ & 14229 & 18.3 & $16.2-20.6$ \\
\hline Extrapulmonary & $837(24.2)$ & 4887 & 13.3 & $10.4-17.0$ \\
\hline Both & $326(9.4)$ & 1652 & 29.7 & $22.4-39.3$ \\
\hline \multicolumn{5}{|l|}{ Sputum Smear } \\
\hline Positive & $1218(35.3)$ & 7150 & 20.6 & $17.5-24.2$ \\
\hline Negative & $1290(37.4)$ & 7426 & 16.3 & 13.6-19.5 \\
\hline Not applicable/missing & $943(27.3)$ & 6191 & 17.1 & $14.2-20.7$ \\
\hline \multicolumn{5}{|l|}{ Chest $x$-ray } \\
\hline Cavitary & $626(18.1)$ & 3769 & 15.1 & 11.7-19.6 \\
\hline Non-cavitary & $2237(64.8)$ & 13107 & 20.0 & $17.7-22.6$ \\
\hline Unknown & $588(17.0)$ & 3891 & 14.1 & 10.9-18.4 \\
\hline \multicolumn{5}{|l|}{ Isoniazid susceptibility } \\
\hline Resistant & $281(8.1)$ & 1713 & 11.7 & 7.5-18.1 \\
\hline Susceptible & $2529(73.3)$ & 14856 & 20.1 & $17.9-22.5$ \\
\hline Unknown & 641 (18.6) & 4199 & 13.3 & $10.3-17.3$ \\
\hline \multicolumn{5}{|l|}{ Streptomycin susceptibility } \\
\hline Resistant & $241(7.0)$ & 1432 & 7.0 & $3.8-13.0$ \\
\hline Susceptible & $2530(73.3)$ & 15062 & 20.2 & $18.0-22.6$ \\
\hline Unknown & $680(19.7)$ & 4274 & 14.0 & $10.9-18.1$ \\
\hline \multicolumn{5}{|l|}{ Previous TB } \\
\hline No & $3168(91.8)$ & 19245 & 17.3 & $15.5-19.2$ \\
\hline Yes & $211(6.1)$ & 1210 & 27.3 & $19.4-38.4$ \\
\hline Unknown & $72(2.1)$ & 313 & 28.8 & $15.0-55.3$ \\
\hline \multicolumn{5}{|l|}{ Excess alcohol use } \\
\hline No & $2924(84.7)$ & 17558 & 16.3 & $14.6-18.4$ \\
\hline Yes & $346(10.0)$ & 1849 & 29.7 & $22.8-38.7$ \\
\hline Unknown & $181(5.2)$ & 1360 & 23.5 & $16.6-33.3$ \\
\hline
\end{tabular}


Table 1 Person-years, deaths and death rates by selected baseline characteristics (Continued)

\begin{tabular}{|c|c|c|c|c|}
\hline \multicolumn{5}{|l|}{ Injection drug use } \\
\hline No & $3090(89.5)$ & 19108 & 17.5 & $15.8-19.5$ \\
\hline Yes & $170(4.9)$ & 395 & 32.9 & $19.1-56.7$ \\
\hline Unknown & $191(5.5)$ & 1264 & 20.6 & $14.0-30.2$ \\
\hline \multicolumn{5}{|l|}{ Correctional facility } \\
\hline No & $3373(97.7)$ & 20240 & 18.1 & $16.3-20.0$ \\
\hline Yes & $93(2.7)$ & 397 & 15.1 & $6.8-33.6$ \\
\hline \multicolumn{5}{|l|}{ Homeless } \\
\hline No & $3073(89.0)$ & 18790 & 17.0 & $15.3-19.0$ \\
\hline Yes & $352(10.2)$ & 1788 & 28.0 & $21.2-36.9$ \\
\hline Unknown & $26(0.8)$ & 170 & 17.7 & $5.7-54.7$ \\
\hline \multicolumn{5}{|l|}{ Long-term care } \\
\hline No & $3358(97.3)$ & 20341 & 17.1 & $15.4-19.0$ \\
\hline Yes & $93(2.7)$ & 397 & 65.2 & $44.4-95.8$ \\
\hline \multicolumn{5}{|l|}{ Therapy type } \\
\hline Directly observed therapy & $2116(61.3)$ & 11794 & 19.2 & $16.8-21.8$ \\
\hline Self-administered therapy & $854(24.7)$ & 6596 & 17.7 & $14.8-21.3$ \\
\hline Both & $391(11.3)$ & 1977 & 11.6 & $7.7-17.5$ \\
\hline Unknown & $90(2.6)$ & 401 & 20.0 & $10.0-39.9$ \\
\hline \multicolumn{5}{|l|}{ Health care provider type } \\
\hline Health department & $1864(54.0)$ & 11727 & 10.3 & $8.6-12.3$ \\
\hline Private & $656(19.0)$ & 4081 & 32.6 & $27.5-38.6$ \\
\hline Both & $880(25.5)$ & 4681 & 24.4 & $20.3-29.3$ \\
\hline Unknown & $51(1.5)$ & 206 & 21.5 & $9.7-47.9$ \\
\hline \multicolumn{5}{|l|}{ Immigration status } \\
\hline U.S.-born & $1283(37.2)$ & 7546 & 34.5 & $30.5-38.9$ \\
\hline Foreign-born & $2168(62.8)$ & & & \\
\hline U.S. resident $\geq 2$ years & $1208(55.7)$ & 14332 & 24.5 & $22.1-27.2$ \\
\hline U.S. resident $<2$ years & $960(44.3)$ & 6436 & 3.6 & $2.4-5.4$ \\
\hline
\end{tabular}

through a private provider and the use of DOT was present only for the 12 month period following TB treatment initiation.

Age, HIV-coinfection and male gender have been previously identified as risk factors for death in subjects diagnosed with TB [7]. To our knowledge, U.S. birth, and recent immigration have not been previously described in relation to survival. U.S. birth has previously been reported as associated with relapse and recurrence of TB [18]. In general, longer life expectancy among immigrant groups has been observed in many types of epidemiologic studies and may relate to a "healthy immigrant effect" or healthier behaviors after immigration compared to U.S -born individuals [19]. A measure of disease severity, site of TB involvement (i.e. miliary/meningeal), was associated with poorer survival in bivariate analysis but did not remain so after adjustment for HIV status.

We found an association of decreased survival and TB treatment from a private provider during the first year following treatment initiation for $\mathrm{TB}$ and this relationship remained when evaluating only those patients that experienced a TB related death. In our model, an individual who received TB treatment from a private provider and survived one year would no longer be at increased risk of death based on this variable. An association between increased physician experience in treating TB and improved survival was recently reported in a Canadian study [11]. Previous studies have observed that management of TB by non-public health providers is associated with inappropriate treatment regimens and lower likelihood of cure. $(24,25)$ In our cohort, one indicator of quality of TB care, documentation of sputum culture conversion in patients with pulmonary TB that completed treatment differed between health department (93\%) and private providers (67\%); similarly HIV status was assessed in $64 \%$ of patients treated by a health department and $52 \%$ of patients treated by private providers ( $\mathrm{p}$-value for both $<0.001$ ).

DOT has been associated with improved likelihood of completion of therapy, decreased relapse, and decreased mortality [11,20-22]. However, a recent study from Spain also found an association between the use of DOT and increased mortality; the authors hypothesized 
Table 2 All-cause mortality using proportional hazards model including time stratified effects for income, provider, and therapy

\begin{tabular}{|c|c|c|c|}
\hline Variable & $\mathrm{HR}$ & $95 \% \mathrm{Cl}$ & $\mathbf{p}$ \\
\hline Age (year) & 1.05 & $1.05,1.06$ & $<0.001$ \\
\hline Male & 1.5 & $1.2,2.0$ & 0.001 \\
\hline \multicolumn{4}{|l|}{ Race/ethnicity } \\
\hline Asian & Reference & -——- & 一一一- \\
\hline Black & 0.7 & $0.4,1.2$ & 0.22 \\
\hline Hispanic & 0.9 & $0.5,1.4$ & 0.56 \\
\hline Native American & 1.1 & $0.6,2.0$ & 0.70 \\
\hline White & 1.2 & $0.8,1.8$ & 0.45 \\
\hline U.S. Born & 1.7 & $1.2,2.5$ & 0.008 \\
\hline $\begin{array}{l}\text { Foreign-born, U.S. resident }<2 \\
\text { years* }\end{array}$ & 0.4 & $0.3,0.7$ & $<0.001$ \\
\hline HIV-positivet & 4.2 & $2.8,6.2$ & $<0.001$ \\
\hline \multicolumn{4}{|l|}{ Major site of Disease } \\
\hline Pulmonary & Reference & -———- & -——- \\
\hline Lymphatic & 1.0 & $0.6,1.5$ & 0.82 \\
\hline $\begin{array}{l}\text { Bone or joint/Genitourinary/ } \\
\text { Peritoneal }\end{array}$ & 1.1 & $0.7,1.7$ & 0.62 \\
\hline Miliary/meningeal & 1.3 & $0.8,2.1$ & 0.31 \\
\hline Other & 0.7 & $0.3,1.7$ & 0.48 \\
\hline Radiographic cavitary disease & 1.1 & $0.8,1.6$ & 0.44 \\
\hline \multicolumn{4}{|l|}{ Drug susceptibility\| } \\
\hline INH susceptible & 1.0 & $0.6,1.7$ & 0.99 \\
\hline Streptomycin susceptible & 1.7 & $0.8,3.7$ & 0.18 \\
\hline Previous history of TB & 1.0 & $0.7,1.6$ & 0.84 \\
\hline Alcohol abuse & 1.2 & $0.8,1.7$ & 0.41 \\
\hline Intravenous drug use & 1.4 & $0.8,2.2$ & 0.24 \\
\hline Homelessness & 0.9 & $0.6,1.2$ & 0.41 \\
\hline Long-term care resident & 1.1 & $0.7,1.7$ & 0.76 \\
\hline \multicolumn{4}{|l|}{ Income $(\$ 5000)$} \\
\hline First year & 0.9 & $0.9,1.0$ & 0.05 \\
\hline After first year & 1.0 & $0.9,1.0$ & 0.25 \\
\hline \multicolumn{4}{|l|}{ Private provider only } \\
\hline Within 1 year of TB diagnosis & 5.1 & $3.5,7.3$ & $<0.001$ \\
\hline $\begin{array}{l}\text { More than } 1 \text { year after TB } \\
\text { diagnosis }\end{array}$ & 1.1 & $0.7,1.5$ & 0.77 \\
\hline \multicolumn{4}{|l|}{ Directly observed therapy } \\
\hline Within 1 year of TB diagnosis & 3.0 & $1.9,4.5$ & $<0.001$ \\
\hline $\begin{array}{l}\text { More than } 1 \text { year after TB } \\
\text { diagnosis }\end{array}$ & 1.0 & $0.7,1.4$ & 0.94 \\
\hline Year of diagnosis after 1999 & 1.0 & $0.8,1.3$ & 0.97 \\
\hline
\end{tabular}

*Reference is immigration more than 2 years prior to TB diagnosis tReference category is HIV-negative or unknown

ICompared to INH and streptomycin susceptible isolates, respectively. Other resistance patterns not included in the model due to limited numbers of isolates.

that DOT may have been preferentially used in complicated patients [23]. We modelled an interaction between health care provider type and therapy type to evaluate our findings. The use of DOT by health departments in this model was not significantly associated with differences in survival. However, we did find an association
Table 3 All-cause mortality using proportional hazards model including directly observed therapy and provider type interaction terms

\begin{tabular}{|c|c|c|}
\hline Variable & HR & $95 \% \mathrm{Cl}$ \\
\hline Age & 1.05 & $1.05,1.06$ \\
\hline Male & 1.5 & $1.2,2.0$ \\
\hline HIV-positive & 4.2 & $2.9,6.3$ \\
\hline \multicolumn{3}{|l|}{ Provider type $\times$ DOT $(\leq 1$ year) } \\
\hline Private provider, no DOT & 2.2 & $1.1,4.6$ \\
\hline Health department, DOT & 1.4 & $0.7,2.8$ \\
\hline Private provider, DOT & 8.5 & $4.3,17.1$ \\
\hline \multicolumn{3}{|l|}{ Provider type $\times$ DOT $\left(>1\right.$ year) ${ }^{*}$} \\
\hline Private provider, no DOT & 1.1 & $0.7,1.7$ \\
\hline Health department, DOT & 1.0 & $0.7,1.5$ \\
\hline Private provider, DOT & 1.0 & $0.5,2.0$ \\
\hline Recent immigrant & 0.4 & $0.3,0.7$ \\
\hline Not foreign born & 1.7 & $1.2,2.5$ \\
\hline
\end{tabular}

* Reference group is health department or both, no DOT

Except for interaction results, only statistically significant $(p<0.05)$ effects are reported. The model was additionally adjusted for race, income, major site of disease, INH susceptibility, streptomycin susceptibility, previous TB, excess alcohol use, excess drug use, homelessness, in long term care, cavitary disease, and year of diagnosis before or after 1999.

between TB medical care by private physicians and decreased survival regardless of the therapy type (i.e. DOT or self-administered), although this association is much stronger in patients who received DOT or combination therapy. A potential explanation for this may be an indication bias where DOT is utilized by private providers based on increased severity of illness or decreased social support. In bivariate analyses, we found increased use of DOT among patients with smear-positive sputum ( $84 \%$ vs. $78 \%$, p-value 0.001$)$, cavitary disease $(83 \%$ vs. $78 \%$, p-value 0.005 ), the homeless ( $96 \%$ vs. $72 \%$, p-value $<0.001)$ and lower incomes $(\mathrm{p}<0.001)$.

There are limitations to our study. First, we were limited in our ability to address potential confounding, particularly related to differences in severity of illness. The observed association between health care provider type and survival could be confounded by differences in severity of illness. We attempted to grade TB severity by major organ site of involvement and the presence of radiographic cavitations. In addition, we performed a sensitivity analysis to address potential confounding due to differences in severity of illness by excluding deaths within 30 days of TB treatment initiation. Second, survival follow-up was passive and our results could also be influenced by incomplete ascertainment of deaths. A disproportionate loss of subjects following the completion of treatment for TB who received their care through a public health department could create the appearance of decreased survival among subjects treated by private health care providers. We simulated plausible differences in follow-up to assess how this would induce 
an artificial association with survival. Our results suggest that it is extremely unlikely that the hazard ratio we observed was due in any large part to differential loss to follow-up. (see additional file 1). Finally, our study was based in a single state in which there is a low prevalence of multidrug-resistant TB and may not apply to other regions with higher prevalence of drug resistance.

Despite these limitations, we believe that this study has important strengths. It is a population-based study that reflects nationwide TB trends. Although we used allcause mortality as the primary outcome of our analysis, similar to other studies of survival following a diagnosis of TB, our observed associations persisted when we limited the outcome to TB related deaths $[7,11,24]$. Our findings, particularly related to associations between provider type and survival, need to be verified in studies where potential confounders can be assessed. If the findings are verified, this would support further efforts to identify mechanisms for the observed differences and work to eliminate disparities in survival. Finally, it is important to emphasize that our findings regarding the use of DOT are likely due to differences in the types of patients recommended for DOT in our study population.

\section{Conclusions}

In conclusion, we identified associations between decreased survival in TB patients and age, male gender, HIV-coinfection, US birth, remote immigration, DOT, and TB treatment by a private health care provider. Although it is important to acknowledge the potential for unmeasured confounding, our study suggests that additional studies are needed to assess associations between TB-related physician experience and TB treatment outcomes.

\section{Additional material}

Additional file 1: Differential loss to follow-up simulation. A

simulation study to assess the potential impact of differential loss to follow-up among subjects treated by private providers as compared to health departments.

Additional file 2: Tuberculosis-related mortality using proportional hazards model including time stratified effects for income, provider, and therapy. We assessed the effect on the model of limiting mortality to TB-related deaths, defined as a TB-related International Classification of Diseases code listed under the multiple contributing causes of death on the death certificate.

Additional file 3: Results of sensitivity analysis after excluding subjects who died within the first $\mathbf{3 0}$ days. As differences in illness severity could introduce confounding if a subject's death and TB diagnosis occurred during the same hospitalization, we assessed the effects on our model of excluding subjects who died within 30-days of a diagnosis of TB.

Additional file 4: Results of analysis evaluating an association between sputum smear status and survival. We evaluated the association of sputum smear positivity and survival in patients with pulmonary TB.

\section{Acknowledgements}

The authors thank Kim Field, RN, MSN TB Control Manager, Washington State Department of Health for her assistance, and the Firland Foundation for its support. DJH was supported by an NRSA grant from the National Institutes of Health.

\section{Author details}

${ }^{1}$ Department of Medicine, University of Washington School of Medicine, Seattle, WA, USA. ${ }^{2}$ Group Health Center for Health Studies, Seattle, WA, USA. ${ }^{3}$ Department of Biostatistics, University of Washington School of Public Health, Seattle, WA, USA. ${ }^{4}$ Department of Epidemiology, School of Public Health, University of Washington, Seattle, WA, USA. ${ }^{5}$ Public Health - Seattle \& King County, Tuberculosis Control Program, Seattle, WA, USA. 'Washington State Department of Health, Infectious Disease and Reproductive Health Assessment Unit, Olympia, WA, USA.

\section{Authors' contributions}

DJH participated in study design, data acquisition, statistical analysis, data interpretation, drafting of the manuscript, and critical revision of the manuscript. RH participated in statistical analysis, data interpretation, drafting of the manuscript, and critical revision of the manuscript. MN participated in data acquisition and critical revision of the manuscript. AE participated in data acquisition, data interpretation, and critical revision of the manuscript. DRP participated in study design and critical revision of the manuscript. CHG participated in study design, statistical analysis, data interpretation, drafting of the manuscript, and critical revision of the manuscript. All authors read and approved the final manuscript.

\section{Competing interests}

The authors declare that they have no competing interests.

Received: 7 April 2010 Accepted: 27 August 2010

Published: 27 August 2010

\section{References}

1. Global Tuberculosis Programme: Global tuberculosis control: WHO report. Geneva: Global Tuberculosis Programme World Health Organization 2009, vol. Began with 1997.

2. Dye C, Scheele S, Dolin P, Pathania V, Raviglione MC: Consensus statement. Global burden of tuberculosis: estimated incidence, prevalence, and mortality by country. WHO Global Surveillance and Monitoring Project. JAMA 1999, 282(7):677-686.

3. Fielder JF, Chaulk CP, Dalvi M, Gachuhi R, Comstock GW, Sterling TR: A high tuberculosis case-fatality rate in a setting of effective tuberculosis control: implications for acceptable treatment success rates. Int I Tuberc Lung Dis 2002, 6(12):1114-1117.

4. Chiang CY, Enarson DA, Yu MC, Bai KJ, Huang RM, Hsu CJ, Suo J, Lin TP: Outcome of pulmonary multidrug-resistant tuberculosis: a 6-yr follow-up study. Eur Respir J 2006, 28(5):980-985.

5. Chan ED, Laurel V, Strand MJ, Chan JF, Huynh ML, Goble M, Iseman MD: Treatment and outcome analysis of 205 patients with multidrugresistant tuberculosis. Am J Respir Crit Care Med 2004, 169(10):1103-1109.

6. van der Sande MA, Schim van der Loeff MF, Bennett RC, Dowling M, Aveika AA, Togun TO, Sabally S, Jeffries D, Adegbola RA, Sarge-Njie R, et al: Incidence of tuberculosis and survival after its diagnosis in patients infected with HIV-1 and HIV-2. AIDS 2004, 18(14):1933-1941.

7. Pablos-Mendez A, Sterling TR, Frieden TR: The relationship between delayed or incomplete treatment and all-cause mortality in patients with tuberculosis. JAMA 1996, 276(15):1223-1228.

8. Sterling TR, Zhao Z, Khan A, Chaisson RE, Schluger N, Mangura B, Weiner M, Vernon A: Mortality in a large tuberculosis treatment trial: modifiable and non-modifiable risk factors. Int I Tuberc Lung Dis 2006, 10(5):542-549.

9. Anyama N, Bracebridge S, Black C, Niggebrugge A, Griffin SJ: What happens to people diagnosed with tuberculosis? A population-based cohort. Epidemiol Infect 2007, 135(7):1069-1076. 
10. Low S, Ang LW, Cutter J, James L, Chee CB, Wang YT, Chew SK: Mortality among tuberculosis patients on treatment in Singapore. Int J Tuberc Lung Dis 2009, 13(3):328-334.

11. Khan K, Campbell A, Wallington T, Gardam M: The impact of physician training and experience on the survival of patients with active tuberculosis. CMAJ 2006, 175(7):749-753.

12. [http://wonder.cdc.gov/tb.html].

13. Initial therapy for tuberculosis in the era of multidrug resistance. Recommendations of the Advisory Council for the Elimination of Tuberculosis. MMWR Recomm Rep 1993, 42(RR-7):1-8.

14. Horne DJ, Hubbard R, Narita M, Exarchos A, Goss CH: Survival following a diagnosis of tuberculosis in Washington State. Am J Respir Crit Care Med 2009, 179:A5281.

15. Curtis $A B$ ME, McKenna $M$, et al: Completeness and timeliness of tuberculosis case reporting, a multistate study. Am J Prev Med 2001, 20:108-112.

16. American Thoracic Society/Centers for Disease Control and Prevention/ Infectious Diseases Society of America: controlling tuberculosis in the United States. Am J Respir Crit Care Med 2005, 172(9):1169-1227.

17. TIMS User's Guide, Appendix SUR I - RVCT Form Completion Instructions. 2003 [http://wonder.cdc.gov/wonder/help/TB/ RVCTFormCompletionlnstructions.pdf].

18. Driver CR, Munsiff SS, Li J, Kundamal N, Osahan SS: Relapse in persons treated for drug-susceptible tuberculosis in a population with high coinfection with human immunodeficiency virus in New York City. Clin Infect Dis 2001, 33(10):1762-1769.

19. Singh GK MB: Health, life expectancy, and mortality patterns among immigrant populations in the United States. Canadian J Pub Health 2004, 95:114-21.

20. Weis SE, Slocum PC, Blais FX, King B, Nunn M, Matney GB, Gomez E, Foresman $\mathrm{BH}$ : The effect of directly observed therapy on the rates of drug resistance and relapse in tuberculosis. N Engl J Med 1994, 330(17):1179-1184.

21. Jasmer RM, Seaman CB, Gonzalez LC, Kawamura LM, Osmond DH, Daley CL: Tuberculosis treatment outcomes: directly observed therapy compared with self-administered therapy. Am J Respir Crit Care Med 2004, 170(5):561-566.

22. Kamolratanakul $P$, Sawert $H$, Lertmaharit $S$, Kasetjaroen $Y$, Akksilp $S$, Tulaporn C, Punnachest K, Na-Songkhla S, Payanandana V: Randomized controlled trial of directly observed treatment (DOT) for patients with pulmonary tuberculosis in Thailand. Transactions of the Royal Society of Tropical Medicine and Hygiene 1999, 93(5):552-557.

23. Cayla JA, Rodrigo T, Ruiz-Manzano J, Caminero JA, Vidal R, Garcia JM, Blanquer R, Casals M: Tuberculosis treatment adherence and fatality in Spain. Respir Res 2009, 10:121.

24. Coady SA, Sorlie PD, Cooper LS, Folsom AR, Rosamond WD, Conwill DE: Validation of death certificate diagnosis for coronary heart disease: the Atherosclerosis Risk in Communities (ARIC) Study. J Clin Epidemiol 2001, 54(1):40-50.

\section{Pre-publication history}

The pre-publication history for this paper can be accessed here: http://www.biomedcentral.com/1471-2334/10/258/prepub

\section{doi:10.1186/1471-2334-10-258}

Cite this article as: Horne et al:: Factors associated with mortality in patients with tuberculosis. BMC Infectious Diseases 2010 10:258.

\section{Submit your next manuscript to BioMed Central and take full advantage of:}

- Convenient online submission

- Thorough peer review

- No space constraints or color figure charges

- Immediate publication on acceptance

- Inclusion in PubMed, CAS, Scopus and Google Scholar

- Research which is freely available for redistribution

Submit your manuscript at www.biomedcentral.com/submit
Biomed Central 